Post-print of journal article: A. Tassone, G. Caruso, F. Giannetti, and A. Del Nevo, "MHD mixed convection flow in the WCLL : Heat transfer analysis and cooling system optimization," Fusion Engineering and Design, 2019. doi: $10.1016 / j$.fusengdes . 2019.01.087 


\title{
MHD mixed convection flow in the WCLL: heat transfer analysis and cooling system optimization
}

\author{
Alessandro Tassone ${ }^{1 *}$, Gianfranco Caruso $^{1}$, Fabio Giannetti ${ }^{1}$, Alessandro Del Nevo ${ }^{2}$ \\ ${ }^{1}$ DIAEE Nuclear Section - Sapienza University of Rome, Corso Vittorio Emanuele II, 244, 00186, Roma, Italy \\ ${ }^{2}$ ENEA FSN-ING-PAN, ENEA CR Brasimone, 40032, Camugnano (BO), Italy
}

\begin{abstract}
In the Water-Cooled Lithium Lead (WCLL) blanket, a critical problem faced by the design is to ensure that the breeding zone (BZ) is properly cooled to avoid the loss of mechanical properties in the structural materials. CFD simulations are performed using ANSYS CFX to assess the cooling system performances accounting for the magnetic field effect in the sub-channel closest to the first wall $(\mathrm{FW})$. Here, intense buoyancy forces $\left(\mathrm{Gr} \approx 10^{10}\right)$ interact with the pressure-driven flow $\left(\mathrm{Re} \approx 10^{3}\right)$ in a MHD mixed convection regime. A constant magnetic field, parallel to the toroidal direction, is assumed with $\mathrm{Ha}=8550$. The walls bounding the channel and the water pipes are modeled as perfectly conducting. The magnetic field is found to dampen the velocity fluctuations triggered by the buoyancy forces and the flow is similar to a forced convection regime. The PbLi heat transfer coefficient is reduced to one-third of its ordinary hydrodynamic value and, consequently, hot-spots close to the $\mathrm{FW}$ are observed, where $T_{\mathrm{Max}} \approx 1000 \mathrm{~K}$. Optimization strategies for the BZ cooling system layout are proposed and implemented in the CFD model, thus fulfilling the design criterion.
\end{abstract}

Keywords: Magnetohydrodynamics (MHD), blanket engineering, WCLL, mixed convection, CFD

\section{Introduction}

The Water Cooled Lithium-Lead (WCLL) blanket is under development as a candidate for implementation in the DEMO fusion reactor [1]. In the WCLL, Lithium Lead $(\mathrm{PbLi})$ is employed as breeder, pressurized water at 15.5 $\mathrm{MPa}$ as first wall (FW) and breding zone (BZ) coolant, and Eurofer steel as structural material. A single module segmentation approach is adopted with the blanket being segmented only in the toroidal direction [2]. To preserve the Eurofer mechanical properties, the steel bulk and interface temperature must not exceed $823 \mathrm{~K}\left(550{ }^{\circ} \mathrm{C}\right)$ during normal operation [3]. Four alternative configurations are currently being studied to identify advantages and key issues, in order to select the reference configuration that will be further developed in the next years.

The magnetohydrodynamic (MHD) effect on the performances is a key design issue for liquid metal blankets. Transition to the MHD regime is accompanied by severe increase in pressure drop, flow stabilization and heat transfer degradation [4]. To limit the pressure drop, the WCLL minimizes the PbLi velocity and uses a non-electrically conductive fluid as coolant. Due to this strategy, the intense temperature gradient fostered by the neutronic power deposition generates buoyancy forces that add to the main

\footnotetext{
*Corresponding author:

Email address: alessandro.tassone@uniroma1.it (Alessandro Tassone $^{1}$ )
}

forced convection flow, thus generating in the BZ a MHD mixed convection regime. Previous thermal-hydraulic studies have reported that buoyancy forces are fundamental in shaping the temperature distribution in the WCLL [5]. The rationale for this study is to evaluate the BZ cooling system performances when the magnetic field is applied and the role played by magneto-convection.

\section{Problem formulation}

For this study, one of the four configurations proposed for the WCLL outboard blanket is considered [6]. The BZ is occupied by long rectangular channels that run all along the blanket poloidal height with the breeder flowing upward inside them. The blanket cross-section assumes the appearance of a checkerboard composed by 4 (radial $) \times 8$ (toroidal $)$ channels. To ensure the BZ refrigeration, double-walled pipes are inserted horizontally from the back part of the segment through openings drilled in the toroidal-poloidal stiffening plates. Two nested U-pipes constitute the elementary component of the BZ cooling system, which is responsible for the refrigeration of a 4-channel radial stack spanning from FW to the back plate. This cooling element is then repeated uniformly for the channel poloidal extension. Therefore, the pitch $\left(p_{v}\right)$ between cooling elements is a characterizing parameter for the blanket layout.

The flow in the channel close to the FW is simulated to demonstrate that the BZ cooling system can effectively 


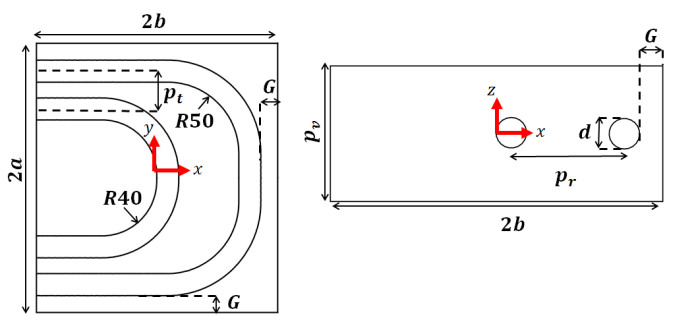

Fig. 1. Cell geometry: radial-toroidal (left), radial-poloidal (right)

Table 1

Channel geometry parameters, length in $(\mathrm{mm})[6]$

\begin{tabular}{lclc}
\hline Parameter (Symbol) & Value & Parameter (Symbol) & Value \\
\hline Toroidal half-length $(a)$ & 82 & Vertical pitch $\left(p_{v}\right)$ & 60 \\
Radial half-length $(b)$ & 73.5 & Radial pitch $\left(p_{r}\right)$ & 50 \\
Pipe wall thickness $\left(t_{w}\right)$ & 2.75 & Toroidal pitch $\left(p_{t}\right)$ & 23 \\
Pipe ext. diameter $\left(d_{o}\right)$ & 13.5 & Pipe-wall distance $(G)$ & 10.25 \\
Pipe int. diameter $\left(d_{i}\right)$ & 8 & Eq. diameter $\left(d_{H}\right)$ & 76 \\
\hline
\end{tabular}

refrigerate the elementary cell and the structural materials: for this purpose, we choose to enforce a conservative condition on the maximum $\mathrm{PbLi}$ temperature in the cell, i.e. $T_{\text {Max }} \leq 823 \mathrm{~K}$. An overview of the problem geometry is available in Figure 1, whereas the main geometrical parameters are collected in Table 1 . The elementary cell volume and equivalent hydraulic diameter are defined as $V=4 a b p_{v}$ and $d_{H}=4 a b /(a+b)$.

The governing equations for a steady, induction-less (i.e. low magnetic Reynolds number), incompressible and laminar MHD flow are obtained by the combination of the Navier-Stokes' and Maxwell's sets. Choosing the electric potential formulation and modeling the buoyancy forces with the Boussinesq's approximation [7], they can be written in the dimensionless form, employing the scales proposed by Bühler [8], as following

$$
\begin{gathered}
\nabla \cdot \vec{v}=0 \\
L y^{-2}(\vec{v} \cdot \nabla) \vec{v}=-\nabla p_{d}+H a^{-2} \nabla^{2} \vec{v}+\vec{J} \times \vec{B}-\vec{g} T \\
P e(\vec{v} \cdot \nabla) T=\nabla^{2} T+Q \\
\vec{j}=-\nabla \phi+\vec{v} \times \vec{B} \\
\nabla \cdot \vec{j}=0
\end{gathered}
$$

Combining the Ohm's law (4) and charge conservation (5), it is found the Poisson equation

$$
\nabla^{2} \phi=\nabla \cdot(\vec{v} \times \vec{B})
$$

which, once solved, provides the electric potential $\phi$ and, through (4), the current density $\vec{j}$. The symbols $Q, \vec{B}$, and $p_{d}$ represent, respectively, the volumetric power source, the applied magnetic field, and the dynamic pressure, which is obtained by the normalized difference from the local pressure and the isothermal hydrostatic pressure at the reference temperature [8]. The gravity is defined as
Table 2

Dimensionless parameters at $T_{\text {ref }}=710 \mathrm{~K}$

\begin{tabular}{lcc}
\hline Parameter & Symbol & Value \\
\hline Grashof number & $\mathrm{Gr}=g \rho^{2} \beta b^{3} \Delta T / \mu^{2}$ & $5.7 \cdot 10^{10}$ \\
Hartmann number & $\mathrm{Ha}=B a(\sigma / \mu)^{0.5}$ & 8550 \\
Lykoudis number & $\mathrm{Ly}=\mathrm{Ha}^{2} / \mathrm{Gr}^{0.5}$ & 306 \\
Peclet number & $\mathrm{Pe}=\mathrm{PrGr}^{2} \mathrm{Ha}^{2}$ & 12.5 \\
Prandtl number & $\mathrm{Pr}=c_{p} \mu / \kappa$ & 0.016 \\
Reynolds number $\left(u_{0} / u_{\mathrm{nc}}\right)$ & $\mathrm{Re}=\rho u_{i} d_{H} / \mu$ & $2038 / 5.6 \cdot 10^{5}$ \\
Richardson number & $\mathrm{Ri}=\mathrm{Gr} / \mathrm{Re}^{2}$ & $1.37 \cdot 10^{4}$ \\
Wall conductance ratio & $\mathrm{c}_{\mathrm{w}}=\sigma_{\mathrm{w}} \mathrm{t}_{\mathrm{w}} / \sigma \mathrm{a}$ & $\infty$ \\
\hline
\end{tabular}

$\vec{g}=-g \hat{z}$. The volumetric heating decreases exponentially moving away from the first wall following the law $Q(x)=(6.5844) \cdot e^{8.8605 x} \mathrm{MW} / \mathrm{m}^{3}$, where $x=[-b, b]$ is the radial coordinate, as described by Martelli et al. [5]. The magnetic field is uniform, constant, and aligned with the toroidal direction, such that $\vec{B}=B \hat{y}$ with $B=4.4 \mathrm{~T}$.

In Table 2, the dimensionless parameters governing the flow features of the considered case are collected. To avoid the build-up of tritium inventory in the blanket, the $\mathrm{PbLi}$ in the channel is compelled to move upward in the poloidal direction ( $z$-axis) with a mass flow rate $\Gamma=0.431 \mathrm{~kg} / \mathrm{s}$, equivalent to a mean velocity $u_{0}=1.825$ $\mathrm{mm} / \mathrm{s}[2,6]$. From the average volumetric power, $\bar{Q}=$ $1 / V \int_{V} Q(x) d V \approx 6.7 \mathrm{MW} / \mathrm{m}^{3}$, it is possible to define a characteristic temperature difference $\Delta T=\bar{Q} b^{2} / \kappa$ and, through the Grashof number, the characteristic velocity for the natural convection contribution is estimated as $u_{n c}=$ $\sqrt{G r} \mu / \rho b=451 \mathrm{~mm} / \mathrm{s}$. Thus, in ordinary hydrodynamic (OHD) conditions, the PbLi flow will be dominated by the natural convection term $(\mathrm{Ri} \gg 1)$ and be turbulent.

In the MHD regime, the square of Ha represents the ratio between electromagnetic and viscous forces, thus, for $\mathrm{Ha} \gg 1$, the latter ones are confined in boundary layers of thickness $\mathrm{O}\left(\mathrm{Ha}^{-1}\right)$ and $\mathrm{O}\left(\mathrm{Ha}^{-1 / 2}\right)$, respectively attached to walls perpendicular or parallel to the magnetic field [7]. The relative ratio between electromagnetic and buoyancy forces is expressed by Ly; if $\gg 1$, the magnetic field dampens the velocity oscillations triggered by the temperature gradient and the inertial term in (2) can be neglected [8]. For the case considered, the characteristic velocity scale for the magneto-convection is defined as $u_{m c}=\mathrm{Gr} / \mathrm{Ha}^{2} \times \mu / \rho b \approx 1.47 \mathrm{~mm} / \mathrm{s}$ and, since $u_{m c} \approx u_{0}$, the forced convection must be modeled. The resulting MHD mixed convection flow in the WCLL channel is expected to be steady, laminar, and inertia-less for the momentum balance, whereas the convection term must be kept in (3), due to $\mathrm{Pe}>1$.

On the duct and pipe walls, the boundary conditions are no-slip $(\vec{v}=0)$ for the velocity and ground $(\phi=0)$ for the electric potential. The ground condition is equivalent to perfectly conducting walls $\left(c_{\mathrm{w}}=\infty\right)$, which it is not a realistic assumption, since in the WCLL blanket both duct and pipe walls will feature finite electrical conductivity, but it is conservative with regard to the resistive Lorentz 
Table 3

PbLi physical properties: implemented temperature dependent correlations and constant values (evaluated at $T_{\text {ref }}=710 \mathrm{~K}$ ) [9]

\begin{tabular}{lll}
\hline Property (unit) & & Value \\
\hline Density $\left(\mathrm{kg} / \mathrm{m}^{3}\right)$ & $\rho$ & 9675.21 \\
Expansion coefficent $(1 / \mathrm{K})$ & $\beta$ & $1.23 \cdot 10^{-4}$ \\
Specific heat $(\mathrm{J} / \mathrm{kgK})$ & $c_{p}$ & 188.49 \\
Permeability $(\mathrm{H} / \mathrm{m})$ & $\mu_{0}$ & $4 \pi \cdot 10^{-7}$ \\
Dynamic viscosity $(\mathrm{Pa} \cdot \mathrm{s})$ & $\mu$ & $1.87 \cdot 10^{-4} e^{(1400 / T)}$ \\
Th. conductivity $(\mathrm{W} / \mathrm{mK})$ & $\kappa$ & $1.95+1.95 \cdot 10^{-2} T$ \\
El. conductivity $(\mathrm{S} / \mathrm{m})$ & $\sigma$ & $\left(1.02 \cdot 10^{-2}+4.26 \cdot 10^{-6} T\right)^{-1}$ \\
\hline
\end{tabular}
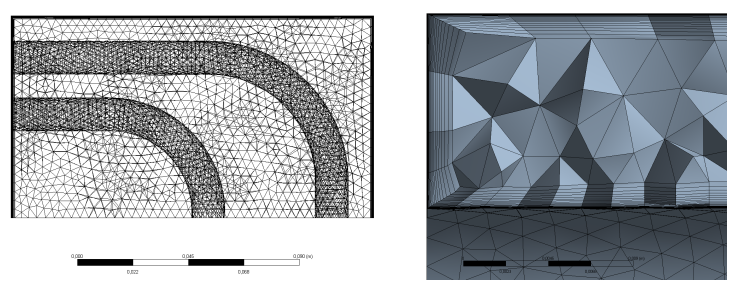

Fig. 2. Mesh and detail of the boundary layer refinement

force experienced by the fluid and, consequently, the heat transfer PbLi-side. The thermal condition is the adiabatic one $(\partial T / \partial n=0)$ for the duct walls and the fixed heat flux $\left(\vec{n} \cdot \nabla T=-q_{w}\right)$ for the pipe, where $\vec{n}$ is the wall inward unit normal. Please note that the heat flux is not uniform on the pipe surface, as described in Section 3. On the top and bottom surfaces, the translational periodicity is imposed to model the long poloidal channel to which the elementary cell belongs: an interface model imposing an upward mass flow rate equal to $\Gamma$ crossing the coupled surfaces is used to represent the momentum source from forced convection.

\section{Numerical model}

Due to the temperature range foreseen in the model, i.e. $T=600 \div 825 \mathrm{~K}$, the temperature dependence is preserved for the PbLi physical properties for which a deviation $\Delta \Phi\left(T_{\mathrm{Max}}, T_{\mathrm{Min}}\right)> \pm 5 \%$ is foreseen with respect to the value at $T_{\text {ref }}=710 \mathrm{~K}$ (see Table 3 for a detailed implementation overview).

The computational domain, shown in Figure 1, is filled with $\mathrm{PbLi}$ initially at rest and at the uniform temperature $T=600 \mathrm{~K}$. The duct walls are at ground potential and adiabatic, therefore their influence on the flow features is completely described by the boundary conditions. Regarding the pipe, an Heat Transfer Coefficient (HTC) model is implemented to calculate the wall heat flux, exempting from the direct simulation of the water-side heat transfer, through the expression $q_{w}=h_{\text {ext }}\left(T_{\text {ext }}-T_{w}\right)$ [10]. Here, $T_{w}$ is the wall temperature, a priori unknown, whereas $h_{\text {ext }}$ and $T_{\text {ext }}$ are the characteristic HTC and bulk temperature of the coolant. Regarding the latter parameter, the midpoint temperature of the coolant thermodynamic cycle $\left(T_{\text {ext }}=584.65 \mathrm{~K}\right)$ is chosen due to the channel position. The HTC is calculated with $h_{\text {ext }}=\left(1 / h_{\text {pipe }}+1 / h_{\mathrm{H} 2 \mathrm{O}}\right)^{-1}=$
$1.05 \cdot 10^{4} \mathrm{~W} / \mathrm{m}^{2} \mathrm{~K}$, accounting for the pipe thermal resistance $\left(h_{\text {pipe }}=2 \kappa_{\mathrm{EU}} / d_{i} \ln \left(d_{o} / d_{i}\right)\right)$ and the water HTC $\left(h_{\mathrm{H} 2 \mathrm{O}}\right)$, obtained from the Dittus-Boelter correlation for turbulent flow in smooth pipes and assuming an average velocity $u_{\mathrm{H} 2 \mathrm{O}}=5 \mathrm{~m} / \mathrm{s}[2]$. The water properties are evaluated at $T_{\text {ext }}$ and $15.5 \mathrm{MPa}$ according to the IAPWS standard [11], as well as the thermal conductivity of the Eurofer from the data reported by Mergia and Boukos [12]. The uncertainty introduced by the HTC model on the wall heat flux was estimated as $\pm 2 \%$ by comparison with a $\mathrm{OHD}$ model implementing the direct simulation of the water-side heat transfer.

To provide a benchmark for the MHD results, the OHD features of the PbLi flow have been investigated by employing a tweaked model. The Shear Stress Transport (SST) turbulence model is used, since it is considered the standard for low Reynolds number simulation and it is mostly insensitive to near-wall resolution, even if $y^{+} \leq 1$ is required for accurate heat transfer modeling [10].

In Figure 2, the unstructred mesh and the boundary layer refinement used to discretize the fluid domain are shown. Since $c_{w}=\infty$, the MHD boundary layer does not carry any current and only 2 nodes are needed to resolve the velocity gradient. Moreover, this layer is very thin, i.e. $\delta_{H}\left(T_{\mathrm{ref}}\right)=\mathrm{O}\left(\mathrm{Ha}^{-1}\right) \approx 10^{-2} \mathrm{~mm}$, so its scale is comparable to the viscous sub-layer that, for free-stream velocity $u_{\infty}=0.2 \mathrm{~m} / \mathrm{s}$, is $\delta\left(y^{+}=1\right) \approx 1.4 \cdot 10^{-2} \mathrm{~mm}$, resulting in $y^{+}=0.17$. Therefore, the computational grid described is suitable for both models. For the OHD simulation, the geometry was extended in the poloidal direction to encompass a 5-cell stack, thus to allow the modeling of vortical structures of length scale $L=p_{v}$. Since the flow is laminar and the water temperature is constant, in the MHD model the PbLi temperature distribution is symmetrical with regard to the $z=0$ plane and a symmetry boundary condition is used to reduce the grid size. The element count for the MHD model is 423'526 with maximum element size $3.5 \mathrm{~mm}$. Maximum and average skewness are reported as 0.797 and 0.187 .

For the time discretization, the time-dependent OHD model adopts a Second Order Backward Euler scheme with average time step $\Delta t=0.015 \mathrm{~s}$, whereas the MHD one uses an High Resolution pseudo-transient scheme with Auto Timescale control for the virtual time step. The simulation reaches the convergence when maximum temperature is constant and the RMS residuals are $\leq 10^{-5}$. Typical run times on 24-core Xeon E5-2690 (2.90 GHz) cluster are about 3 days for the OHD and 1 day for the MHD model.

The MHD model of ANSYS CFX has been validated in the past up to $\mathrm{Ha}=10^{4}$ for pressure-driven and buoyancydriven benchmarks in rectangular ducts for $c_{w}=[0, \infty]$. Further details are reported in [13].

\section{Results and discussion}

In OHD conditions, the flow is dominated by the natural convection. In Figure 3, the maximum velocity observed is 


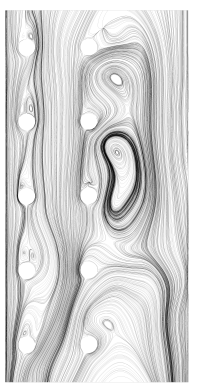

(a) Velocity streamlines

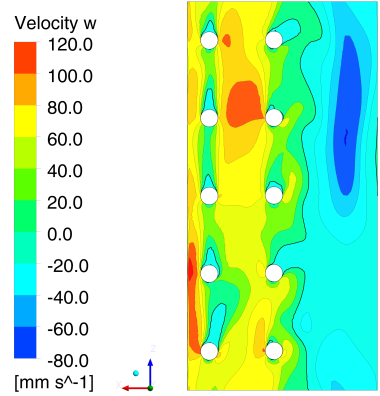

(b) Poloidal velocity contour
Fig. 3. OHD velocity streamlines and contour on the radial-poloidal plane at $y=0$. Thick dark line in Figure 3b identifies the $w=0$ iso-surface.
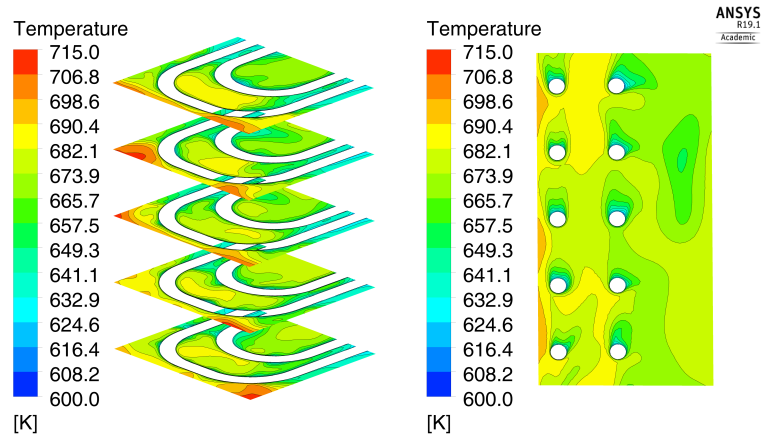

Fig. 4. OHD temperature contour on the $x-y$ planes passing through the pipe centers (left) and the radial-poloidal plane at $y=0$ (right)

$v \approx 120 \mathrm{~mm} / \mathrm{s}$, almost two orders of magnitude higher than the one due to forced convection, and vortices of length scale equal to $p_{v}$ appear between the cooling elements. Due to these phenomena, the heat transfer is extremely efficient and the PbLi maximum temperature is well below the threshold $\left(T_{\mathrm{Max}, \mathrm{OHD}}=714 \mathrm{~K}\right)$. In Figure 4 , it can be seen how the temperature is low in the channel back, whereas hotspots are present close to the FW in the duct corners.

When the magnetic field is applied, the flow becomes dominated by Lorentz forces and, due to the high field intensity, it reverts to a laminar and steady regime, with a velocity scale comparable to the forced convection term. The vortices observed in OHD are completely suppressed, although the buoyancy forces still shape the velocity profile, as it can be seen in Figure 5. Characteristic magnetoconvection features, like opposing jets close to the FW and cooling pipes, appear in the front part of the channel, whereas a slug flow dominates the back, where the heating is less intense $[7,8]$.

The heat transfer is dampened and becomes dominated by the conduction mechanism, even if the convection contribution still cannot be neglected, as it is evidenced by the warping upstream of the isotherms in Figure 6, where
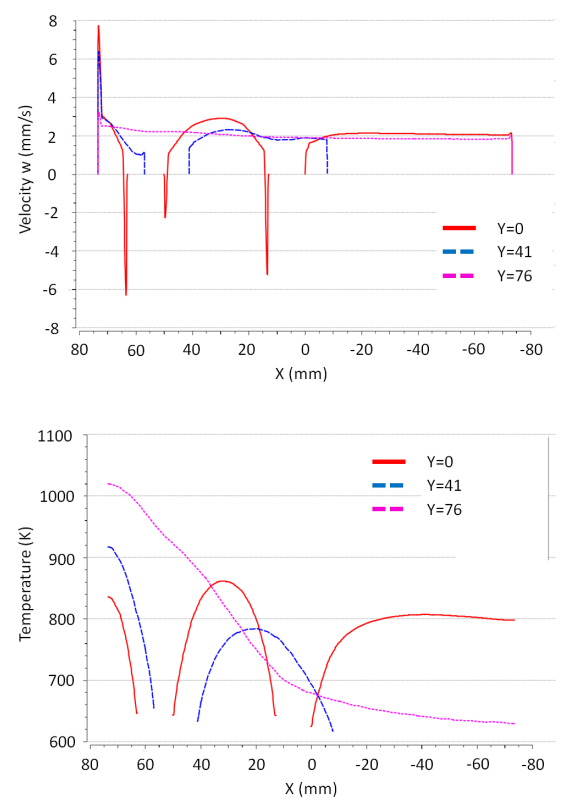

Fig. 5. MHD vertical velocity (top) and temperature profiles (bottom) along the radial direction on the toroidal-radial plane at $z=0$ : cell center $(y=0)$, inner/outer pipe gap $(y=41 \mathrm{~mm})$, and outer pipe/lateral wall $(y=76 \mathrm{~mm})$.

the temperature contour is plotted considering $T=820 \mathrm{~K}$ as the scale ceiling to highlight the regions where the $\mathrm{PbLi}$ is above the chosen temperature threshold. Hotspots are present at the corners and in the radial gap between the pipes. The maximum temperature recorded in the cell is $1031 \mathrm{~K}$ (see Figure 5).

Considering the area-averaged heat flux removed from the pipe surface $\left(\overline{q_{w}}\right)$, the heat transfer coefficient $\left(h_{\mathrm{LM}}\right)$ between $\mathrm{PbLi}$ and pipe surface is evaluated as

$$
h_{\mathrm{LM}}=\left(\bar{T}-T_{\mathrm{ext}} / \overline{q_{w}}-1 / h_{\mathrm{ext}}\right)^{-1}
$$

with $\bar{T}$ the average PbLi temperature calculated on $z=$ 0 plane. It is found that $h_{\mathrm{LM}} \approx 3000 \mathrm{~W} / \mathrm{m}^{2} \mathrm{~K}$ for the MHD case, about one-third of the value calculated in $\mathrm{OHD}$ conditions $\left(\approx 9400 \mathrm{~W} / \mathrm{m}^{2} \mathrm{~K}\right)$.

\subsection{BZ cooling system optimization}

Previous thermo-hydraulic studies have suggested that the FW cooling system passively refrigerate the nearby BZ region removing among $5 \% \div 10 \%$ of the total power deposited with a mean heat flux $q_{\mathrm{FW}}=-130 \mathrm{~kW} / \mathrm{m}^{2}$ [5]. This phenomenon is represented in the CFD model assuming a constant heat flux removed from the FW. The maximum temperature trend with $q_{\mathrm{FW}}$ is shown in Table 4 . Corner hotspots are efficiently cooled by this strategy, but only for the highest flux considered the temperature falls below the threshold.

Successively, the vertical pitch $\left(p_{v}\right)$ is reduced, thus decreasing the amount of power to be removed by the single cooling element. FW passive refrigeration is assumed 


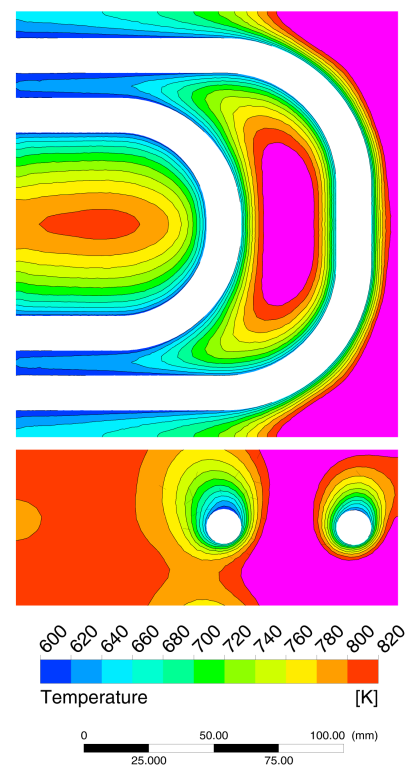

Fig. 6. MHD cell temperature distribution on the plane $z=0$ (top) and $y=0$ (bottom) for $p_{v}=60 \mathrm{~mm}$ and adiabatic $\mathrm{FW}$

Table 4

Maximum temperature in the cell versus optimization parameter. For $p_{v}$ reduction, $q_{F W}=-100 \mathrm{~kW} / \mathrm{m}^{2}$ is assumed

\begin{tabular}{cccc}
\hline \multicolumn{2}{c}{ Passive FW cooling } & \multicolumn{2}{c}{ Vertical pitch reduction } \\
$q_{\mathrm{FW}}\left[\mathrm{kW} / \mathrm{m}^{2}\right]$ & $T_{\mathrm{Max}}$ & $p_{v}[\mathrm{~mm}]$ & $T_{\text {Max }}[\mathrm{K}]$ \\
\hline 0 & 1031 & 60 & 898 \\
-50 & 941 & 55 & 873 \\
-100 & 885 & 50 & 854 \\
-150 & 844 & 45 & 835 \\
-200 & 817 & 40 & 823 \\
\hline
\end{tabular}

at $q_{\mathrm{FW}}=-100 \mathrm{~kW} / \mathrm{m}^{2}$. Maximum temperature trend is reported in Table 4 . For $p_{v}=40 \mathrm{~mm}$, the temperature in the cell is everywhere below the criterion but, as it is possible to observe in Fig. 7, corner hotspots are still present. In absence of $\mathrm{FW}$ refrigeration, even for $p_{v}=$ $40 \mathrm{~mm}, T_{\mathrm{Max}}>900 \mathrm{~K}$.

\section{Conclusions}

A CFD model of the FW channel of the WCLL blanket is realized to assess the $\mathrm{BZ}$ cooling system performances in MHD operative conditions. A configuration with poloidal $\mathrm{PbLi}$ flow and horizontal cross-flow U-pipes for the coolant is considered. The magnetic field suppresses buoyancyinduced velocity oscillations reverting the intense turbulent OHD flow into laminar state. The PbLi-side heat transfer coefficient is reduced to one-third, with conduction becoming the dominant mechanism, and the temperature reaches above $1000 \mathrm{~K}$. The condition $T_{\mathrm{Max}} \leq 823 \mathrm{~K}$ for the $\mathrm{PbLi}$ is met by introducing a moderate amount of passive refrigeration from the $\mathrm{FW}\left(q_{\mathrm{FW}}=-100 \mathrm{~kW} / \mathrm{m}^{2}\right)$ and reducing the distance between cooling elements to $p_{v}=40 \mathrm{~mm}$.

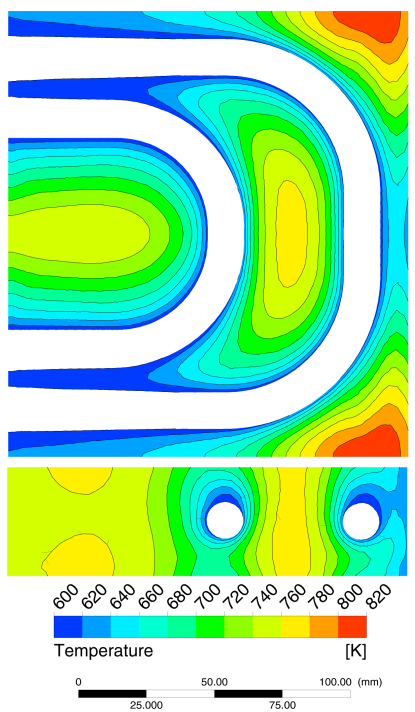

Fig. 7. MHD cell optimized temperature distribution on the plane $z=0$ (top) and $y=0$ (bottom) for $p_{v}=40 \mathrm{~mm}$ and $q_{\mathrm{FW}}=-100$ $\mathrm{kW} / \mathrm{m}^{2}$

Further optimization is possible by modifying the pipe layout, e.g. bringing them closer to the walls, and considering more realistic electromagnetic boundary conditions for the walls. For the latter, increased peak velocities are foreseen close to the cooling pipe according to the asymptotic theory developed by Buhler, which would enhance the heat transfer [7]. A more refined model considering finite conductivity walls and with direct simulation of the FW and BZ cooling system is under development to provide more accurate results.

\section{Acknowledgements}

This work has been carried out within the framework of the EUROfusion Consortium and has received funding from the Euratom research and training programme 20142018 under grant agreement No 633053. The views and opinions expressed herein do not necessarily reflect those of the European Commission.

\section{References}

[1] F. Cismondi, et al., Progress in EU Breeding Blanket design and integration, Fusion Engineering and Design 136 (2018) 782-792.

[2] A. Tassone, et al., Recent progress in the WCLL breeding blanket design for the demo fusion reactor, IEEE Transactions on Plasma Science 46 (2018) 1446-1457.

[3] J. Aubert, G. Aiello, C. Bachmann, P. A. Di Maio, R. Giammusso, A. L. Puma, A. Morin, A. Tincani, Optimization of the first wall for the DEMO water cooled lithium lead blanket, Fusion Engineering and Design 98 (2015) 1206-1210.

[4] S. Smolentsev, et al., MHD thermofluid issues of liquid-metal blankets: phenomena and advances, Fusion Engineering and Design 85 (2010) 1196-1205.

[5] E. Martelli, et al., Thermo-hydraulic analysis of EU DEMO WCLL breeding blanket, Fusion Engineering and Design 130 (2018) 48-55. 
[6] R. Mozzillo, et al., Assessment on DEMO water cooled lithium lead alternative design configuration, Fusion Engineering and Design (2018). (submitted).

[7] U. Müller, L. Bühler, Magnetofluiddynamics in channels and containers, Springer Science \& Business Media, 2001.

[8] L. Bühler, Laminar buoyant magnetohydrodynamic flow in vertical rectangular ducts, Physics of Fluids (1994-present) 10 (1998) 223-236.

[9] U. Jauch, et al., Thermophysical properties in the system Li-Pb, KIT, 1986.

[10] ANSYS, Inc., CFX Solver Modeling Guide, ANSYS, 2018.

[11] W. Wagner, H.-J. Kretzschmar, IAPWS industrial formulation 1997 for the thermodynamic properties of water and steam, IAPWS-IF97 (2008) 7-150.

[12] K. Mergia, N. Boukos, Structural, thermal, electrical and magnetic properties of Eurofer 97 steel, Journal of Nuclear Materials 373 (2008) $1-8$.

[13] A. Tassone, et al., CFD simulation of the magnetohydrodynamic flow inside the WCLL breeding blanket module, Fusion Engineering and Design 124 (2017) 705-709. 